\title{
Sleep disorders in COPD: the forgotten dimension
}

\author{
Walter T. McNicholas' ${ }^{1}$ Johan Verbraecken ${ }^{2}$ and Jose M. Marin ${ }^{3}$
}

\begin{abstract}
Affiliations: 'Pulmonary and Sleep Disorders Unit, St. Vincent's University Hospital, Conway Institute of Biomolecular and Biomedical Research, University College Dublin, Dublin, Ireland. ${ }^{2}$ Dept of Pulmonary Medicine and Multidisciplinary Sleep Disorders Centre, Antwerp University Hospital and University of Antwerp, Antwerp, Belgium. ${ }^{3}$ Respiratory Service, Hospital Universitario Miguel Servet, Zaragoza, Spain.
\end{abstract}

Correspondence: W.T. McNicholas, Pulmonary and Sleep Disorders Unit, St. Vincent's University Hospital, Elm Park, Dublin 4, Ireland. E-mail: walter.mcnicholasवucd.ie

ABSTRACT Sleep in chronic obstructive pulmonary disease (COPD) is commonly associated with oxygen desaturation, which may exceed the degree of desaturation during maximum exercise, both subjectively and objectively impairing sleep quality. The mechanisms of desaturation include hypoventilation and ventilation to perfusion mismatching. The consequences of this desaturation include cardiac arrhythmias, pulmonary hypertension and nocturnal death, especially during acute exacerbations. Coexistence of COPD and obstructive sleep apnoea (OSA), referred to as overlap syndrome, has been estimated to occur in $1 \%$ of the general adult population. Overlap patients have worse sleep-related hypoxaemia and hypercapnia than patients with COPD or OSA alone. OSA has a similar prevalence in COPD as in a general population of similar age, but oxygen desaturation during sleep is more pronounced when the two conditions coexist. Management of sleep-related problems in COPD should particularly focus on minimising sleep disturbance via measures to limit cough and dyspnoea; nocturnal oxygen therapy is not generally indicated for isolated nocturnal hypoxaemia. Treatment with continuous positive airway pressure alleviates hypoxaemia, reduces hospitalisation and pulmonary hypertension, and improves survival.

○

@ERSpublications

Management of sleep-related problems in COPD should aim to minimise sleep disturbance http://ow.ly/mTJL8

\section{Introduction}

Sleep has several effects on breathing, which include changes in central respiratory control, lung mechanics and muscle contractility, that do not have an adverse effect in healthy individuals but may result in significant hypoxaemia and hypercapnia in patients with chronic obstructive pulmonary disease (COPD), particularly during rapid eye movement (REM) sleep [1]. Gas exchange in sleeping normal subjects is largely a consequence of hypoventilation, although ventilation/perfusion $\left(V^{\prime} / Q^{\prime}\right)$ abnormalities may also contribute. In COPD patients this hypoventilation is more pronounced, particularly during REM-sleep, due to a number of factors that include: airflow obstruction, hyperinflation, respiratory muscle dysfunction, blunted ventilatory responses to hypercapnia and/or hypoxia, $V^{\prime} / Q^{\prime}$ mismatching and medications such as loop diuretics and oral steroids. Sleep disturbance is also common in COPD $[2,3]$ and this is likely a consequence of the underlying lung disease, although adverse effects of drug therapy on sleep quality may also contribute. Sleep disturbance is one of the most common symptoms reported by COPD patients, occurring in $\sim 40 \%$ of patients in one large study [4]. These patients have problems initiating or maintaining sleep, and have increased light sleep and reduced REM sleep, frequent sleep stage shifts and micro-arousals. Sleep efficiency is low, in the range of $50-70 \%$, in the majority of this patient population.

Received: May 152013 | Accepted after revision: June 202013

Conflict of interest: None declared.

Provenance: Submitted article, peer reviewed.

Copyright @ERS 2013 
Sleep disturbance likely contributes to the non-specific daytime symptoms of chronic fatigue, lethargy and overall impairment in quality of life described by these patients. Night-time symptoms in COPD patients frequently go unnoticed by physicians and/or are not reported by patients themselves [2].

The present review discusses the pathophysiological basis of sleep and breathing disturbances in patients with COPD, reviews the overlap syndrome where COPD and obstructive sleep apnoea (OSA) coexist, and finally discusses the management of sleep-related disorders in patients with COPD.

\section{Pathophysiology of sleep-related breathing disturbances in COPD \\ Alterations in respiratory mechanics and ventilatory control during sleep related to COPD}

A combination of factors can contribute to disturbances in ventilation and gas exchange during sleep in COPD, relating to accentuated physiological adaptations, such as hypoventilation, which are summarised in figure 1 [5]. People who are hypoxaemic during wakefulness have resting oxygen saturation levels on the steep portion of the oxyhaemoglobin dissociation curve, which can result in disproportionately greater falls in oxygen saturation at night. In addition, $V^{\prime} / Q^{\prime}$ mismatch and a reduced functional residual capacity (FRC) may also play a role in nocturnal hypoxaemia in COPD.

\section{Accentuated physiological hypoventilation due to central respiratory effects}

Ventilatory control in patients with COPD follows the same basic principles as in normal subjects. During all sleep stages, respiratory centre responses to chemical and other inputs are diminished and respiratory muscle responses to respiratory centre outputs are also diminished, particularly during REM sleep, especially those involving accessory muscles of respiration [6, 7]. During REM sleep, in healthy individuals, ventilation may be $40 \%$ lower than during wakefulness. This is predominantly caused by a reduction in tidal volume, to which increased upper airway resistance and diminished inspiratory drive contribute, and results in a slight fall in arterial oxygen saturation $\left(\mathrm{SaO}_{2}\right)$ that is not clinically significant in normal subjects [8]. A similar breathing pattern is present during sleep in patients with COPD. However, the physiological hypoventilation normally present during sleep is accentuated, resulting in profound hypoxaemia in patients with respiratory insufficiencies such as COPD [9]. This is likely a result of the increased physiological dead space in COPD, which leads to an even greater decrease in alveolar ventilation with lower tidal volumes than in normal subjects [10]. Because many COPD patients have hypoxaemia while awake, they are especially prone to nocturnal oxygen desaturation as a result of being on the steep portion of the oxyhaemoglobin dissociation curve [1].

In addition, during non-REM sleep, the basal metabolic rate and ventilatory drive are decreased. These effects may result in a decreased central respiratory drive in non-REM sleep. In REM sleep, the respiratory centre has a diminished response to chemical and mechanical inputs [11]. Studies performed in COPD patients have demonstrated that a lower response of minute ventilation to $\mathrm{CO}_{2}$ can already be present during wakefulness. However, the response measured from parameters that are less influenced by respiratory mechanics, such as mouth occlusion pressure $0.1 \mathrm{~s}$ after onset of inspiration ( $\mathrm{P}_{0.1}$ ), appear to be less impaired, if not normal [5]. Thus, although the receptor and respiratory centre response to hypercapnia may be normal, this response cannot be translated into ventilation due to peripheral mechanisms relating to airflow obstruction, in addition to the hyperinflation and/or respiratory muscle dysfunction affecting such patients $[11,12]$.

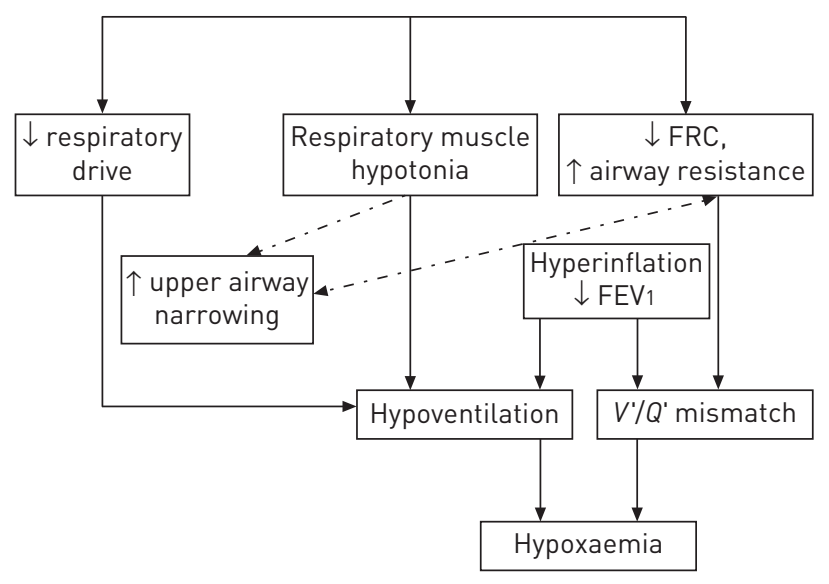

FIGURE 1 Pathophysiology of sleep-related respiratory changes in chronic obstructive pulmonary disease. Sleep has negative effects on various aspects of respiration resulting in worsening hypoxaemia. FRC: functional residual capacity; FEV1: forced expiratory volume in $1 \mathrm{~s}$; $V^{\prime} / Q^{\prime}$ : ventilation/perfusion ratio. 
Altered airway resistance and respiratory muscle contractility

Upper airway resistance increases during sleep compared to wakefulness $[13,14]$ due to a loss of tone in the upper pharyngeal muscles. This may also alter the ventilatory responses to hypoxia and hypercapnia, and may contribute to hypoventilation. Consequently, the resistance of the supraglottic airways increases from wakefulness to sleep to a similar degree in normal subjects as in COPD patients. However, the dilatory response of the supraglottic airways to increases in arterial carbon dioxide tension $\left(\mathrm{PaCO}_{2}\right)$ is significantly lower in COPD patients, which indicates an impaired response of lowering the upper airway resistance, which can further contribute to nocturnal hypercapnia and hypoxaemia [15]. Moreover, there is mild bronchoconstriction during sleep as a feature of the normal circadian change in airway calibre, which may be exaggerated in patients with COPD, thus resulting in an increase in lower airway resistance as well, as previously demonstrated in patients with bronchial asthma [16].

In addition, there is hypotonia of skeletal muscles (tongue, pharyngeal, laryngeal and intercostal muscles) during sleep and a change in the relative contribution of rib cage and abdominal compartment to breathing $[17,18]$. During REM sleep, there is a marked loss of tonic activity in the intercostal muscles, due to supraspinal inhibition of $\gamma$-motoneurons (and to a lesser extent $\alpha$-motoneurons), in addition to presynaptic inhibition of afferent terminals from muscle spindles. The diaphragm, being driven almost entirely by $\alpha$-motoneurons and with far fewer spindles than in the intercostal muscles, has little tonic (postural) activity and, therefore, escapes the reduction of this particular drive during REM sleep [8]. This reduction in accessory respiratory muscle activity during REM sleep may contribute to hypoventilation, especially in COPD patients who are particularly reliant on accessory muscle activity to maintain ventilation. One of the pathological consequences of COPD is stretching of the diaphragm due to lung hyperinflation, which may reduce the efficiency of diaphragmatic contraction, thus necessitating an increased accessory muscle contribution to breathing [19]. Subjects with severe COPD may be totally dependent on a poorly functioning diaphragm for ventilation during sleep [5]. Skeletal muscle atrophy and dysfunction are common in advanced COPD, which may further compromise the contribution made by accessory muscles [20]. Diaphragmatic efficiency is further compromised by the supine position, since the pressure of abdominal contents against the diaphragm exerted by gravitational force contrasts with the effect of gravity in the erect position, which tends to move the abdominal contents away from the diaphragm. Since the diaphragm is virtually the only respiratory muscle that is active during REM sleep, this will lead to paradoxical breathing and further hypoventilation. This probably explains why significant correlations have been observed between variables relating to respiratory muscle strength (maximal inspiratory mouth pressure) and mean nocturnal $\mathrm{SaO}_{2}$ in COPD patients.

\section{Respiratory mechanics during sleep in COPD}

$V^{\prime} / Q^{\prime}$ mismatch in COPD results from progressive airflow limitation and emphysematous destruction of the pulmonary capillary bed. A small, but statistically significant, reduction in FRC occurs during sleep, principally because of a reduction in tonic muscle activity and an increase in airway resistance, which may augment $V^{\prime} / Q^{\prime}$ mismatch [21]. In addition, the supine position is associated with a $10 \%$ decrease in FRC in normal subjects. These physiological changes in pulmonary function, when imposed on subjects with COPD, may adversely affect the $V^{\prime} / Q^{\prime}$ mismatch, by closing small airways in the dependent lung zones [22]. This results in lower oxygen reserves, in addition to lower carbon dioxide buffering capacity, which contributes to nocturnal oxygen desaturation and hypercapnia. Furthermore, cephalad displacement of the diaphragm and a decrease in lung compliance are other possible mechanisms contributing to this reduction in FRC [19]. Evidence for a role of $V^{\prime} / Q^{\prime}$ mismatch in the pathophysiology of nocturnal oxygen desaturation in COPD comes from the observation that $\mathrm{PaCO}_{2}$ levels rise to a similar extent in those patients who develop major nocturnal oxygen desaturation as in those who develop only a minor degree of desaturation, which suggests a similar degree of hypoventilation in both groups [23]. Moreover, cardiac output is maintained during these episodes of hypoventilation, indicating changes in global $V^{\prime} / Q^{\prime}$ matching $[24,25]$.

Worsening of $V^{\prime} / Q^{\prime}$ mismatch during sleep in COPD has also been explained by a decreased mucociliary clearance. $V^{\prime} / Q^{\prime}$ mismatch due to pulmonary emphysema and small airways disease is measurable even in subjects with mild COPD, but appears to increase in line with disease progression. However, there is evidence based on body plethysmographic studies that FRC does not change during sleep in patients with COPD, but these data were only gathered in five patients [26].

\section{Impact of COPD on sleep quality}

Sleep quality is often impaired in patients with COPD, which is likely to be an important factor in the chronic fatigue, sleepiness and overall impairment in quality of life reported by these patients [27, 28]. There is an increased prevalence of insomnia, use of hypnotic medications and an increase in daytime sleepiness in subjects with COPD compared with the general population [29]. KLINK et al. [29] reported that 
$39 \%$ of patients with nocturnal cough or wheezing reported difficulty initiating or maintaining sleep. If cough and wheeze were both present, 53\% reported difficulty initiating or maintaining sleep, and $23 \%$ reported excessive daytime sleepiness [29]. It seems that in patients with mild obstructive airways disease, there is little impact on sleep quality [30]. However, as COPD becomes more severe, there are an increasing number of sleep complaints with possibly more deleterious physiological effects $[3,28]$.

Sleep tends to be fragmented, with frequent arousals and diminished amounts of deep sleep and REM sleep $[3,31]$. KwON et al. [32] reported a strong relationship between hyperinflation and lower sleep efficiency in patients with the overlap syndrome, but the effect was independent of OSA after correction for the apnoea/ hypopnoea index (AHI).

The cause of this poor sleep quality is not entirely clear. The role of hypoxaemia as a respiratory stimulant is rather weak, since quality of sleep is not improved by adding oxygen, and thus, hypercapnia is considered as a much stronger stimulant in provoking arousals [33]. Also, increased inspiratory loads, due to hyperinflation and intrinsic positive end-expiratory pressure, can substantially add to the work of breathing and, hence, elicit arousals by stimulation of mechanoreceptors in the chest wall and lower airways. The arousal response is lowest for hypercapnia and hypoxia during REM sleep, but the arousal response to inspiratory loading is relatively preserved [28]. Nocturnal cough may disturb sleep in these patients, as may the use of drugs like theophylline that are frequently used as a bronchodilator. Last but not least, cigarette smokers manifest disturbances in the sleep electroencephalogram that are not evident in conventional measures of sleep architecture. Nicotine in cigarette smoke and withdrawal from it during sleep may contribute to a higher alpha power and the subjective experience of non-restorative sleep [34]. Unfortunately, sleep impairment is an aspect of COPD that is frequently neglected by many physicians, and in trials designed to assess the impact of COPD on quality of life [2]. Moreover, it has been shown that sleep deprivation is associated with a mild decrease in forced vital capacity (FVC) (-5\%) and forced expiratory volume in $1 \mathrm{~s}$ (FEV1) (-6\%) [35]. When extrapolating these data towards COPD patients with sleep fragmentation, it could be speculated that disturbed sleep quality may lead to worse outcomes in COPD. This was confirmed by OMACHI et al. [36] who demonstrated that disturbed sleep is predictive of COPD exacerbations, emergency healthcare utilisation and mortality. However, ITO et al. [37] proved that depression but not sleep disturbance itself is an independent factor affecting exacerbations and hospitalisation in COPD.

\section{Overlap syndrome of COPD and OSA}

The coexistence of COPD and obstructive sleep apnoea was first described as the "Overlap Syndrome" by David Flenley almost 30 years ago [38]. He pointed out that a sleep study should be considered in obese COPD patients, in those who snore, or those who complain of headache following nocturnal oxygen therapy to determine the presence of associated OSA. He questioned nocturnal oxygen administration in these patients and believed that the clinical course and prognosis of overlap patients was worse than patients suffering from COPD or OSA alone. These opinions remain valid today.

\section{Definitions and epidemiology}

The latest version of the Global Initiative for Chronic Obstructive Lung Disease strategy document defined COPD as a common preventable and treatable disease, characterised by persistent airflow limitation that is usually progressive and associated with an enhanced chronic inflammatory response in the airways and the lung to noxious particles or gases. It underlines that "exacerbations and comorbidities contribute to the overall severity in individual patients" [39]. Clearly, the coexistence of OSA is one of these comorbidities. The diagnosis of COPD should be considered in any patient who has chronic respiratory symptoms, mainly exertional dyspnoea, chronic cough and/or sputum production, and a history of exposure to risk factors for the disease (mainly cigarette smoking). Spirometry is required to make the diagnosis in this clinical context and the presence of a post-bronchodilator $\mathrm{FEV} 1 / \mathrm{FVC}<0.70$ confirms the presence of persistent airflow limitation and, thus, of COPD.

At sleep onset, patients with OSA suffer recurrent pharyngeal collapse and temporary cessation of breathing (apnoea). Such apnoea events cause repetitive hypoxia and carbon dioxide retention, and provoke awakenings (i.e. arousals) that restore airflow. However, once sleep resumes, pharyngeal obstruction and subsequent apnoea recurs. An OSA disorder is generally defined as an AHI $>5$ events $\cdot \mathrm{h}^{-1}[40,41]$. Patients may present with daytime and/or nocturnal complaints, but frequently close companions are the first to push for medical attention because of concerns regarding snoring and/or witnessed apnoea. The presence and severity of the sleep disordered breathing is confirmed by a sleep study.

In pulmonary clinics, OSA, COPD and asthma are the most prevalent chronic respiratory disorders. The prevalence of COPD is directly related to the prevalence of tobacco smoking, but $10 \%$ of the general 
population around the world have moderate-to-severe COPD (FEV1/FVC $<0.7$ plus FEV1 $<80 \%$ predicted) [42]. As defined above, the prevalence of OSA was more than $20 \%$ of males and $9 \%$ of females $[43,44]$. Obesity is one of the main risk factors for OSA and obesity rates have risen since these population studies were performed. Therefore, it is logical to think that current rates of prevalence of OSA are much higher.

There are some indirect data about the prevalence of overlap syndrome. In the Sleep Heart Health Study, a large community-based cohort study which included polysomnography and spirometry, $0.5 \%$ of the participants had airflow obstruction [30]. In a European study with predominantly mild COPD patients, OSA occurred in $3 \%$ [45].

\section{Sleep in patients with COPD and OSA}

In a recent European survey, $78.1 \%$ of patients with COPD reported some degree of night-time symptoms [46]. They also reported that as the severity of airflow limitation increased so did the prevalence of nighttime symptoms. Patients reporting bothersome symptoms at night experienced more daytime breathlessness, had more exacerbations within the previous 12 months and were receiving more maintenance medications than those who did not report bothersome night-time symptoms. Unfortunately, in this survey OSA symptoms or sleep studies were not recorded. Therefore, the potential impact of the coexistence of OSA in the night-time symptoms of COPD patients is unknown. The presence of COPD symptoms, such as dyspnoea, cough, sputum or wheezing, is associated with arousals and difficulty with maintenance of sleep. Decreased total sleep time and sleep efficiency have been confirmed in sleep studies [47], which likely contributes to the complaint of daytime hypersomnolence by many patients.

Patients with COPD develop significant nocturnal oxygen desaturation, and daytime gas exchange abnormalities, particularly a low $\mathrm{PaO}_{2}$, are predictive of nocturnal desaturation [23]. Nevertheless, more than $50 \%$ of $\mathrm{COPD}$ patients with daytime $\mathrm{SaO}_{2}>90 \%$ without sleep apnoea experience significant desaturation during sleep [48]. This phenomenon has been largely ignored, although an increased mortality in these patients compared to those who do not desaturate has previously been described.

The prevalence of OSA is not greater in COPD patients compared with the non-COPD population. Nevertheless some predisposing factors such as age, active smoking, peripheral oedema and oral corticosteroids, increase the risk of obstructive apnoea events. Obesity, when present among COPD patients, is a key contributor for sleep disordered breathing, accelerated pulmonary hypertension and obesity hypoventilation syndrome irrespective of airflow obstruction severity [49]. These patients particularly resemble the so-called "blue bloater" COPD phenotype. By contrast, some patients with advanced COPD can lose weight and consequently reduce the risk for upper airway obstruction (fig. 2).

\section{Clinical features of the overlap syndrome}

Nocturnal hypoxaemia is one of the most important sleep abnormalities in COPD and OSA. The overlap syndrome causes more severe nocturnal hypoxaemia than either disease alone (fig. 3). Hypoxaemic events are associated with both systemic and pulmonary increases in blood pressure and arrhythmias [51, 52]. A well-established chronic effect of these events is the development of cor pulmonale. In patients with the overlap syndrome, surrogates of OSA severity such AHI, seem to play a minor role for developing pulmonary hypertension compared with variables that reflect the severity of COPD. For example, daytime hypoxaemia, hypercapnia and reduced FEV1, were found to be predictors of right-heart failure [53].

Nocturnal death appears to increase in COPD exacerbations [54] and OSA compared with non-COPD, non-OSA [55]. No data are available on this issue in the overlap syndrome, although in the report by MCNicholas and FitzGerald [54] nocturnal death was highest amongst "blue-bloater" type patients with type 2 respiratory failure, a COPD phenotype commonly associated with sleep disordered breathing. Overall, the evidence indicates that mortality is increased in overlap patients. In OSA patients studied at sleep clinics, the coexistence of COPD increased risk of death [56]. We have recently confirmed this data in our cohort of patients referred with suspected sleep disordered breathing. In addition to a sleep study all patients underwent spirometry. After a median follow-up of $>9$ years, all-cause mortality was higher in the untreated overlap group (42.2\%) than in the COPD-only group (24.2\%). Comorbid OSA remained a risk factor for death after adjusting for COPD severity [57]. Continuous positive airway pressure (CPAP) was offered to all patients to treat OSA following local guidelines and among those who accepted and adhered to the treatment, CPAP eliminated the additional mortality risk of OSA in overlap patients, compared with the COPD-only patients (fig. 4). In a Brazilian cohort of patients with overlap syndrome and daytime hypoxaemia, CPAP was offered in addition to long-term oxygen therapy (LTOT) [58]. Among those that could not afford the CPAP, did not adhere to or refused treatment, the 5-year survival was only $26 \%$ versus $71 \%$ with CPAP and LTOT, versus $26 \%$ with LTOT alone. In both studies, CPAP was not provided in a randomised, blinded manner, so a definitive conclusion on the protective effect of CPAP among overlap 


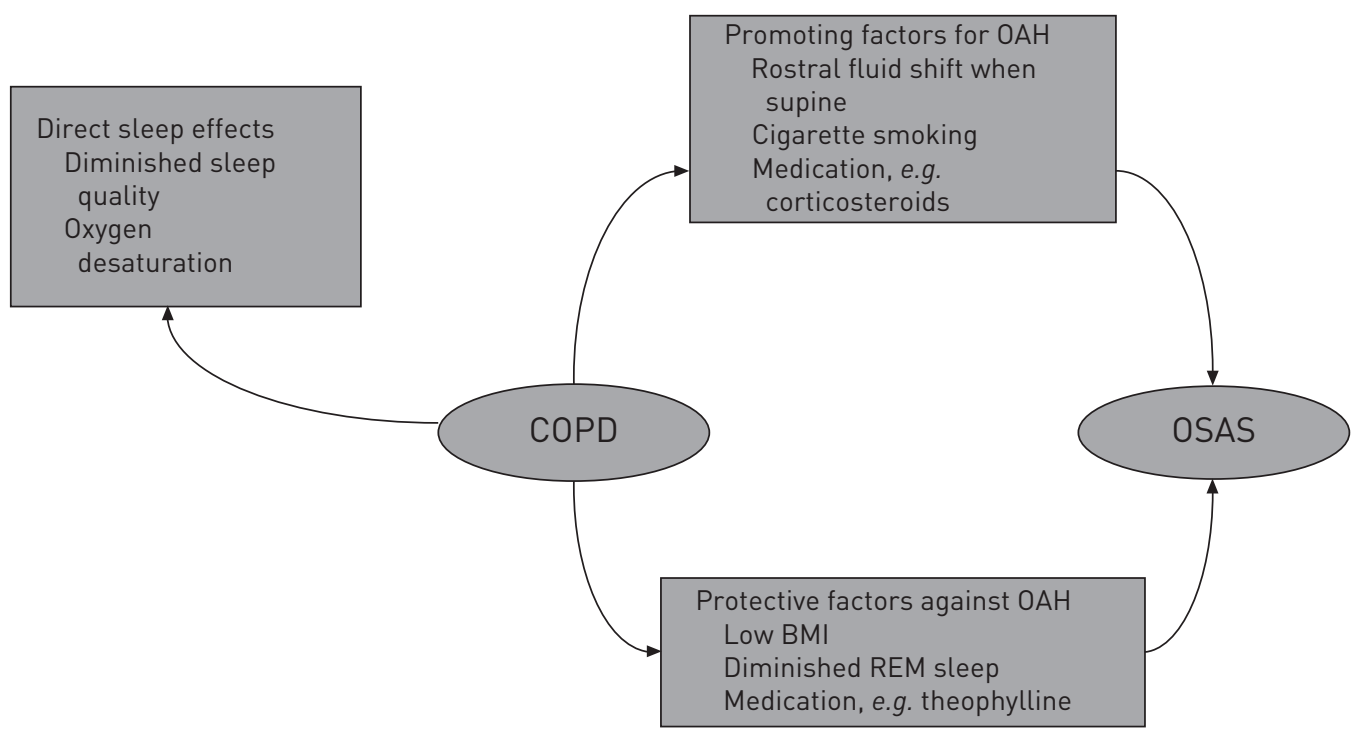

FIGURE 2 Pathophysiological interactions between chronic obstructive pulmonary disease (COPD), sleep and obstructive sleep apnoea syndrome (OSAS). Interactions between COPD, sleep and OSAS are shown, highlighting factors relating to COPD that may promote or inhibit the development of obstructive apnoea and hypopnoea (OAH). BMI: body mass index; REM: rapid eye movement. Reproduced from [50] with permission from the publisher.

patients cannot be given. In our series, death in the untreated overlap group was most commonly attributed to cardiovascular diseases [57]. There is no clear mechanistic explanation for this finding. Since we also found an increase in severe COPD exacerbations in the untreated group, we can speculate that in overlap syndrome the increased incidence of COPD exacerbations may accelerate lung-function decline, which is associated with greater mortality [59].

\section{Management of sleep disorders in COPD}

Since patients with COPD experience disturbed sleep quality in addition to worsening gas exchange during sleep, management should consider each aspect of the disorder. However, the first management principle of sleep-related breathing disturbances in COPD should be to optimise the underlying condition, which will almost invariably benefit breathing while asleep. Correction of hypoxaemia is particularly important and in recent years considerable interest has focussed on the potential benefits of noninvasive ventilation (NIV).

\section{Oxygen therapy}

The most serious consequence of hypoventilation, particularly during sleep, is hypoxaemia, and appropriate oxygen therapy plays an important part in the management of any disorder associated with respiratory insufficiency during sleep. Care must be taken that correction of hypoxaemia is not complicated by hypercapnia in patients with COPD, since respiratory drive in such patients may be partly dependent on the stimulant effect of hypoxaemia. Therefore, the concentration of added oxygen should be carefully titrated to bring the arterial oxygen tension $\left(\mathrm{PaO}_{2}\right)$ up into the mildly hypoxaemic range in order to minimise the tendency towards carbon dioxide retention, particularly during sleep. However, the risk of carbon dioxide retention with supplemental oxygen therapy in such patients may have been overstated in the past, and there is evidence that carbon dioxide retention with oxygen supplementation during sleep is often modest, and usually non-progressive [60]. In particular, there appears to be a low risk of serious carbon dioxide retention with carefully controlled oxygen therapy during exacerbations of COPD even when relatively high flow oxygen supplementation is required to bring the $\mathrm{SaO}_{2}$ into the region of $90-92 \%$ [61]. Thus, the priority in oxygen supplementation should be to provide sufficient oxygen to bring the $\mathrm{SaO}_{2}$ level above $90 \%$, but doing so in a controlled fashion to avoid excessive supplementation. Oxygen supplementation during sleep is best delivered via nasal cannulae, since face masks are more likely to become dislodged during sleep [62].

In the chronic setting, indications for supplemental oxygen are best determined by measures that indicate the overall magnitude of hypoxaemia during sleep, such as the cumulative time spent with $\mathrm{SaO}_{2}<90 \%$. Furthermore, the potential benefits of nocturnal oxygen therapy in patients with moderate daytime hypoxaemia $\left(\mathrm{PaO}_{2} 55-60 \mathrm{mmHg}\right)$ remain unclear and are the subject of ongoing large prospective studies. 


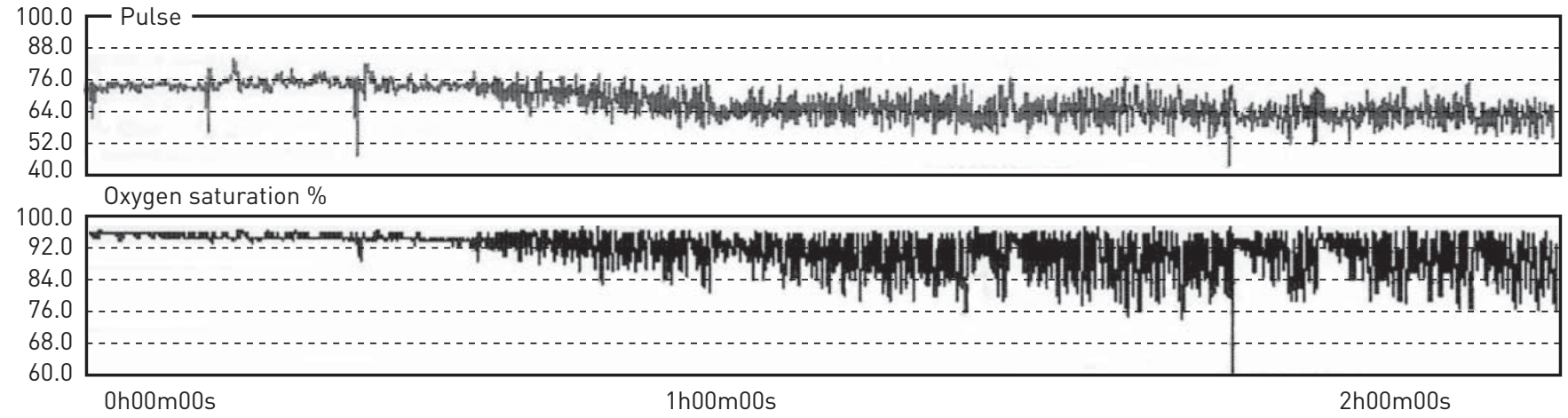

b) 100.0 - Pulse

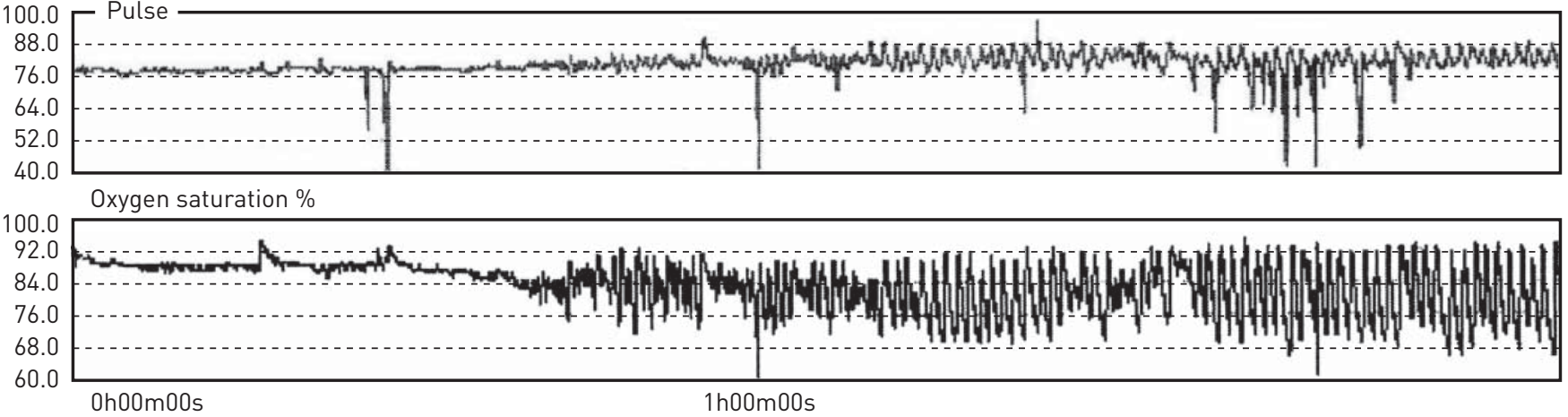

FIGURE 3 Arterial oxygen saturation $\left(\mathrm{SaO}_{2}\right)$ patterns during sleep in obstructive sleep apnoea (OSA) alone and the overlap syndrome. $\mathrm{SaO}_{2}$ patterns in a patient with a) OSA alone and b) overlap syndrome demonstrating the persisting pattern of desaturation in the overlap patient whereas the OSA patient returns to normal $\mathrm{SaO}_{2}$ between apnoea events.

\section{Pharmacological therapy}

Considerations of pharmacological therapy in the setting of sleep in patients with COPD should address the impact of conventional therapeutic agents, such as bronchodilators and anti-inflammatory agents, on sleep and gas exchange, and also the impact on ventilation and gas exchange of pharmacological agents that are designed to improve sleep quality.

\section{Conventional bronchodilator and anti-inflammatory agents used in COPD}

Cholinergic tone is increased at night and it has been proposed that this contributes to airflow obstruction and deterioration in gas exchange during sleep in patients with obstructive airways disease. One report demonstrated significant improvements in both sleep quality and gas exchange in patients with COPD treated with ipratropium [63]. Another study demonstrated significant improvements in nocturnal $\mathrm{SaO}_{2}$ with the once-daily anticholinergic agent, tiotropium, without significant changes in sleep quality [64]. Improvements in $\mathrm{SaO}_{2}$ were particularly significant during REM sleep, which is clinically significant since REM sleep is associated with the most severe oxygen desaturation.

There are only limited data on the efficacy of beta-agonists in the management of sleep-related breathing abnormalities in COPD. However, a recent report demonstrated improvements in gas exchange during sleep with the long-acting beta-agonist salmeterol to a similar degree to that seen with tiotropium [65].

The effects of theophylline have also been studied during sleep in patients with COPD. In addition to being a bronchodilator, theophylline has important effects on respiration that may be particularly beneficial in patients with sleep-related respiratory disturbance, including central respiratory stimulation and improved diaphragmatic contractility [66]. Beneficial effects on $\mathrm{SaO}_{2}$ and arterial carbon dioxide levels in COPD during sleep have also been demonstrated [67]. Theophylline has an added advantage in that there is evidence of beneficial effects in OSA [68]. The mechanism of this effect in COPD appears to be mainly due to a reduction in trapped gas volume rather than bronchodilation. However, the principal limiting effect of theophyllines in this context is an adverse effect on sleep quality, in contrast to the anticholinergic agents, discussed above. There are no data on the potential for newer phosphodiesterase 4 agents, such as rofumilast, to benefit sleep-related variables in COPD. 

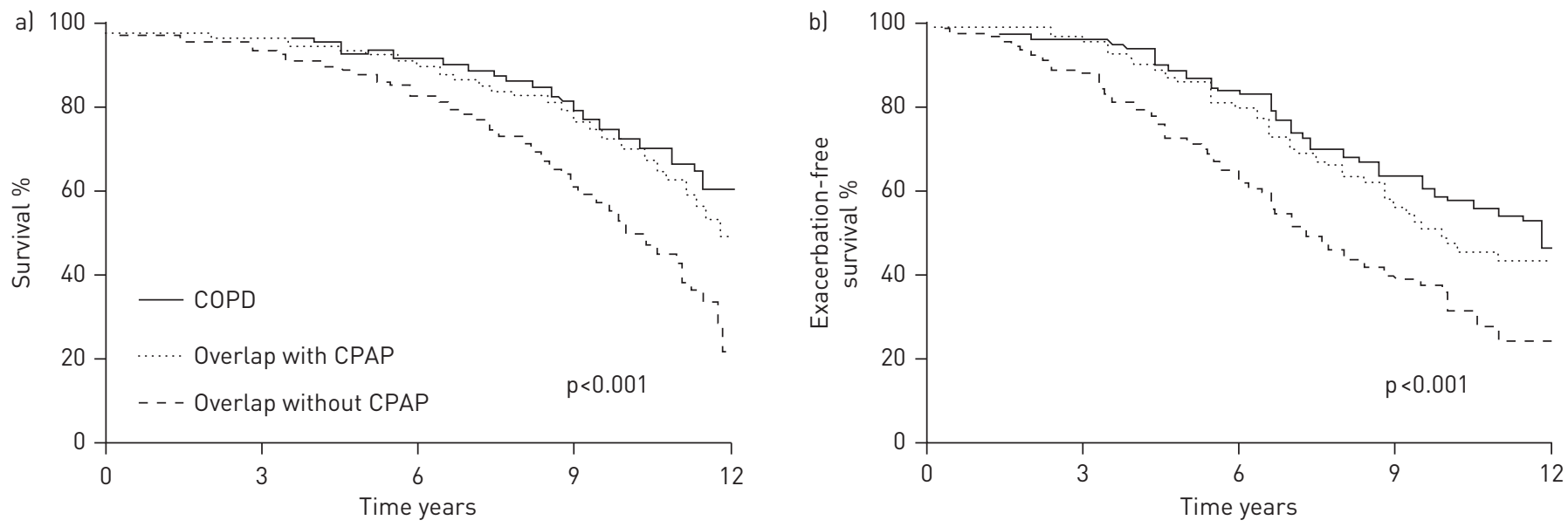

FIGURE 4 Kaplan-Meier survival curves for outcomes among chronic obstructive pulmonary disease (COPD) patients without obstructive sleep apnoea (OSA) (COPD group), patients with COPD and coexisting OSA (overlap group), and patients with overlap syndrome treated with continuous positive airway pressure (CPAP) since enrolment (overlap with CPAP group). a) Survival and b) severe COPD exacerbation-free survival curves among the three study groups. The differences between curves from the COPD only and COPD with OSA treated with CPAP groups are statistically significant from the curve of patients with COPD and untreated OSA $(\mathrm{p}<0.001)$. Reproduced from [57] with permission from the publisher.

\section{Other medications with potential to improve sleep-disordered breathing in COPD}

Historically almitrine was used as a therapy, it is a powerful carotid body agonist that stimulates ventilation and improves ventilation/perfusion relationships within the lung, probably via enhancement of hypoxic pulmonary vasoconstriction. The overall effect is to lessen hypoxaemia, which could potentially be a useful addition in the management of conditions associated with nocturnal hypoxaemia, particularly COPD. Significant improvements in nocturnal $\mathrm{SaO}_{2}$ have been reported compared with placebo, which were most pronounced during REM sleep [69]. However, important side-effects include pulmonary hypertension, dyspnoea and peripheral neuropathy, which resulted in almitrine being withdrawn and the agent is no longer available.

Tricyclic antidepressants and selective serotonin reuptake inhibitors have also been studied in the context of sleep disordered breathing in COPD. These agents produce a fragmentation of REM sleep, and thus, may reduce the severity of oxygen desaturation in this sleep stage. There appears to be a short-term benefit to nocturnal $\mathrm{SaO}_{2}$ levels in COPD [70], although this benefit may not persist with long-term use of the drug [71]. Therefore, despite its theoretical role, this agent is rarely used in the management of sleep-related breathing disturbances in COPD.

\section{Therapies used to promote sleep}

Benzodiazepine and non-benzodiazepine hypnotics shorten sleep latency, improve sleep efficiency and decrease arousal frequency, but have adverse effects on ventilation that include hypoventilation with associated hypoxaemia and hypercapnia, in addition to diminished arousal response to hypercapnia and increased apnoea frequency [72]. Thus, these agents should be avoided, if possible, in patients with severe COPD, although there is evidence that some hypnotics, such as zolpidem, can be used in less severe COPD without significant adverse effects on gas exchange [73].

Melatonin receptor antagonists, such as ramelteon, also shorten sleep latency and improve sleep efficiency, and have been reported to have no adverse effects on apnoea frequency or $\mathrm{PaO}_{2}$ levels in patients with COPD [74]. There is also evidence that cognitive behavioural therapy for insomnia in COPD results in positive sleep and fatigue effects [75].

\section{Noninvasive pressure support}

Patients with COPD associated with respiratory insufficiency who fail to respond to pharmacological therapy should be considered for some form of assisted ventilation. In an acute setting, this may require intubation and ventilation, but in the past two decades increasing attention has been directed towards noninvasive methods of ventilatory support, particularly during sleep $[76,77]$. NIV is particularly indicated in patients with hypercapnic respiratory failure. Long-term nocturnal NIV can also be considered in COPD patients with chronic respiratory failure where improvements in gas exchange during wakefulness have been reported [78], in addition to improvements in respiratory muscle strength and endurance [79]. Sleep quality and diurnal $\mathrm{PaO}_{2}$ and $\mathrm{PaCO}_{2}$ levels are better with NIV plus supplemental oxygen than with 
supplemental oxygen alone [80]. Several mechanisms are likely to play a role in these improvements, including the resting of chronically fatigued respiratory muscles thereby improving daytime respiratory muscle function [81]. Lung compliance is also improved by reversing microatelectasis and preventing collapse of the airways leading to a reduction in the work of breathing. Furthermore, over time, home mechanical ventilation is thought to lead to resetting of the chemoreceptor drive to breathe, as a direct result of reversal of nocturnal hypoventilation [78]. Patients with severe awake blood gas derangement appear to tolerate NIV relatively well. Although long-term NIV therapy is associated with sustained improvements in waking gas exchange, improvements in long-term survival have not been clearly documented [82-87].

Patients with the overlap syndrome should also be treated by nocturnal pressure support and the choice between CPAP or bilevel positive airway pressure can be determined based on the pattern of sleep disordered breathing. In cases where OSA predominates, CPAP may be most appropriate, whereas in cases where there is evidence of significant nocturnal hypoventilation with associated periods of sustained hypoxaemia, bilevel positive airway pressure may be more appropriate. Newer modalities of pressure support, such as adaptive servo ventilation, may be particularly suited to patients with the overlap syndrome.

\section{Conclusion}

Patients with COPD are subject to a range of sleep-related abnormalities that include poor sleep quality and sleep disordered breathing with associated hypoxaemia. Thus, clinical attention should be given to sleeprelated features in these patients, which represent underexplored potential for improvements in the clinical features and quality of life among these patients.

\section{References}

McNicholas WT. Impact of sleep in COPD. Chest 2000; 117: Suppl. 2 48S-53S.

2 Agusti A, Hedner J, Marin JM, et al. Night-time symptoms: a forgotten dimension of COPD. Eur Respir Rev 2011; 20: 183-194.

3 McSharry DG, Ryan S, Calverley P, et al. Sleep quality in chronic obstructive pulmonary disease. Respirology 2012; 17: 1119-1124.

4 Rennard S, Decramer M, Calverley PMA, et al. Impact of COPD in North America and Europe in 2000: subjects' perspective of Confronting COPD International Survey. Eur Respir J 2002; 20: 799-805. McNicholas WT. Impact of sleep in respiratory failure. Eur Respir J 1997; 10: 920-933.

Douglas NJ, White DP, Pickett CK, et al. Respiration during sleep in normal man. Thorax 1982; 37: 840-844.

Tusiewicz K, Moldofsky H, Bryan AC, et al. Mechanics of the rib cage and diaphragm during sleep. J Appl Physiol 1977; 43: 600-602.

$8 \quad$ Phillipson E. Control of breathing during sleep. Am Rev Respir Dis 1978; 118: 909-939.

9 Douglas NJ, Calverley PM, Leggett RJ, et al. Transient hypoxaemia during sleep in chronic bronchitis and emphysema. Lancet 1979; 313: 1-4.

10 Becker HF, Piper AJ, Flynn WE, et al. Breathing during sleep in patients with nocturnal desaturation. Am J Respir Crit Care Med 1999; 159: 112-118.

11 Scano G, Spinelli A, Duranti R, et al. Carbon dioxide responsiveness in COPD patients with and without chronic hypercapnia. Eur Respir J 1995; 8: 78-85.

12 Kent BD, McNicholas WT, Verbraecken J. Disturbed sleep and COPD outcomes: cart meets horse. Sleep Med 2011; 13: 453-454.

13 Doherty LS, Nolan P, McNicholas WT. Effects of topical anesthesia on upper airway resistance during wake-sleep transitions. J Appl Physiol 2005; 99: 549-555.

14 Wiegand L, Zwillich CW, White DP. Collapsibility of the human upper airway during normal sleep. J Appl Physiol 1989; 66: 1800-1808.

15 Meurice JC, Marc I, Sériès F. Influence of sleep on ventilatory and upper airway response to $\mathrm{CO}_{2}$ in normal subjects and patients with COPD. Am J Respir Crit Care Med 1995; 152: 1620-1626.

16 Hetzel MR, Clark TJH. Comparison of normal and asthmatic circadian rhythms in peak expiratory flow rate. Thorax 1980; 35: 732-738.

17 Tabachnik E, Muller NL, Bryan AC, et al. Changes in ventilation and chest wall mechanics during sleep in normal adolescents. J Appl Physiol 1981; 51: 557-564.

18 Millman RP, Knight H, Kline LR, et al. Changes in compartmental ventilation in association with eye movements during REM sleep. J Appl Physiol 1988; 65: 1196-1202.

19 Johnson MW, Remmers JE. Accessory muscle activity during sleep in chronic obstructive pulmonary disease. J Appl Physiol 1984; 57: 1011-1017.

20 Engelen MPKJ, Schols AMWJ, Does JD, et al. Skeletal muscle weakness is associated with wasting of extremity fatfree mass but not with airflow obstruction in patients with chronic obstructive pulmonary disease. Am J Clin Nutr 2000; 71: 733-738.

21 Hudgel DW, Devadatta P. Decrease in functional residual capacity during sleep in normal humans. J Appl Physiol 1984; 57: 1319-1322.

22 Hudgel DW, Martin RJ, Capehart M, et al. Contribution of hypoventilation to sleep oxygen desaturation in chronic obstructive pulmonary disease. J Appl Physiol 1983; 55: 669-677.

23 Mulloy E, McNicholas WT. Ventilation and gas exchange during sleep and exercise in severe COPD. Chest 1996; 109: 387-394.

24 Catterall JR, Calverley PM, MacNee W, et al. Mechanism of transient nocturnal hypoxemia in hypoxic chronic bronchitis and emphysema. J Appl Physiol 1985; 59: 1698-1703. 


\section{8; 114: 958-964.}

Es, 151.945

Sceresink S, et al. Perception of fatigue and quality of life in patients with COPD. Chest

28 Cormick W, Olson LG, Hensley MJ, et al. Nocturnal hypoxaemia and quality of sleep in patients with chronic obstructive lung disease. Thorax 1986; 41: 846-854.

29 Klink M, Quan SF. Prevalence of reported sleep disturbances in a general adult population and their relationship to obstructive airways diseases. Chest 1987; 91: 540-546.

30 Sanders MH, Newman AB, Haggerty CL, et al. Sleep and sleep-disordered breathing in adults with predominantly mild obstructive airway disease. Am J Respir Crit Care Med 2003; 167: 7-14.

31 Saaresranta T, Irjala K, Aittokallio T, et al. Sleep quality, daytime sleepiness and fasting insulin levels in women with chronic obstructive pulmonary disease. Respir Med 2005; 99: 856-863.

32 Kwon JS, Wolfe LF, Lu BS, et al. Hyperinflation is associated with lower sleep efficiency in COPD with co-existent obstructive sleep apnea. COPD 2009; 6: 441-445.

33 Hedemark LL, Kronenberg RS. Ventilatory and heart rate responses to hypoxia and hypercapnia during sleep in adults. J Appl Physiol 1982; 53: 307-312.

34 Zhang L, Samet J, Caffo B, et al. Power spectral analysis of EEG activity during sleep in cigarette smokers. Chest 2008; 133: 427-432.

35 Phillips BA, Cooper KR, Burke TV. The effect of sleep loss on breathing in chronic obstructive pulmonary disease. Chest 1987; 91: 29-32.

36 Omachi TA, Blanc PD, Claman DM, et al. Disturbed sleep among COPD patients is longitudinally associated with mortality and adverse COPD outcomes. Sleep Med 2012; 13: 476-483.

37 Ito K, Kawayama $\mathrm{T}$, Shoji $\mathrm{Y}$, et al. Depression, but not sleep disorder, is an independent factor affecting exacerbations and hospitalization in patients with chronic obstructive pulmonary disease. Respirology 2012; 17: 940-949.

38 Flenley DC. Sleep in chronic obstructive lung disease. Clin Chest Med 1985; 6: 651-661.

39 Vestbo J, Hurd SS, Agustí AG, et al. Global strategy for the diagnosis, management, and prevention of chronic obstructive pulmonary disease. Am J Respir Crit Care Med 2013; 187: 347-365.

40 Sleep-related breathing disorders in adults: recommendations for syndrome definition and measurement techniques in clinical research. The report of an American Academy of Sleep Medicine Task Force. Sleep 1999; 22: 667-689.

41 McNicholas WT. Diagnosis of obstructive sleep apnea in adults. Proc Am Thorac Soc 2008; 5: $154-160$.

42 Buist AS, McBurnie MA, Vollmer WM, et al. International variation in the prevalence of COPD (the BOLD study): a population-based prevalence study. Lancet 2007; 370: 741-750.

43 Durán J, Esnaola S, Rubio R, et al. Obstructive sleep apnea-hypopnea and related clinical features in a populationbased sample of subjects aged 30 to 70 yr. Am J Respir Crit Care Med 2001; 163: 685-689.

44 Young T, Palta M, Dempsey J, et al. The occurrence of sleep-disordered breathing among middle-aged adults. N Engl J Med 1993; 328: 1230-1235.

45 Bednarek M, Plywaczewski R, Jonczak L, et al. There is no relationship between chronic obstructive pulmonary disease and obstructive sleep apnea syndrome: a population study. Respiration 2005; 72: 142-149.

46 Price D, Small M, Milligan G. The prevalence and impact of night-time symptoms in COPD patients - results of a cross-sectional study in five European countries. Proc of the IV World Asthma and COPD Forum 2011.

47 Krachman SL, Chatila W, Martin UJ, et al. Effects of lung volume reduction surgery on sleep quality and nocturnal gas exchange in patients with severe emphysema. Chest 2005; 128: 3221-3228.

48 Lewis CA, Fergusson W, Eaton T, et al. Isolated nocturnal desaturation in COPD: prevalence and impact on quality of life and sleep. Thorax 2009; 64: 133-138.

49 Chaouat A, Bugnet AS, Kadaoui N, et al. Severe pulmonary hypertension and chronic obstructive pulmonary disease. Am J Respir Crit Care Med 2005; 172: 189-194.

50 McNicholas WT. Chronic obstructive pulmonary disease and obstructive sleep apnoea: overlaps in pathophysiology, systemic inflammation, and cardiovascular disease. Am J Respir Crit Care Med 2009; 180: 692-700.

51 Tirlapur VG, Mir MA. Nocturnal hypoxemia and associated electrocardiographic changes in patients with chronic obstructive airways disease. N Engl J Med 1982; 306: 125-130.

52 Chaouat A, Naeije R, Weitzenblum E. Pulmonary hypertension in COPD. Eur Respir J 2008; 32: 1371-1385.

53 Chaouat A, Weitzenblum E, Kessler R, et al. Sleep-related $\mathrm{O}_{2}$ desaturation and daytime pulmonary haemodynamics in COPD patients with mild hypoxaemia. Eur Respir J 1997; 10: 1730-1735.

54 McNicholas WT, FitzGerald MX. Nocturnal death among patients with chronic bronchitis and emphysema. BMJ 1984; 289: 878 .

55 Gami AS, Howard DE, Olson EJ, et al. Day-night pattern of sudden death in obstructive sleep apnea. $N$ Engl J Med 2005; 352: 1206-1214.

56 Lavie P, Herer P, Peled R, et al. Mortality in sleep apnea patients: a multivariate analysis of risk factors. Sleep 1995; 18: 149-157.

57 Marin JM, Soriano JB, Carrizo SJ, et al. Outcomes in patients with chronic obstructive pulmonary disease and obstructive sleep apnea: the overlap syndrome. Am J Respir Crit Care Med 2010; 182: 325-331.

58 Machado MCL, Vollmer WM, Togeiro SM, et al. CPAP and survival in moderate-to-severe obstructive sleep apnoea syndrome and hypoxaemic COPD. Eur Respir J 2010; 35: 132-137.

59 Donaldson GC, Seemungal TAR, Bhowmik A, et al. Relationship between exacerbation frequency and lung function decline in chronic obstructive pulmonary disease. Thorax 2002; 57: 847-852.

60 Goldstein RS, Ramcharan V, Bowes G, et al. Effect of supplemental nocturnal oxygen on gas exchange in patients with severe obstructive lung disease. N Engl J Med 1984; 310: 425-429.

61 Moloney ED, Kiely JL, McNicholas WT. Controlled oxygen therapy and carbon dioxide retention during exacerbations of chronic obstructive pulmonary disease. Lancet 2001; 357: 526-528. 
62 Costello RW, Liston R, McNicholas WT. Compliance at night with low flow oxygen therapy: a comparison of nasal cannulae and Venturi face masks. Thorax 1995; 50: 405-406.

63 Martin RJ, Bartelson BL, Smith P, et al. Effect of ipratropium bromide treatment on oxygen saturation and sleep quality in COPD. Chest 1999; 115: 1338-1345.

64 McNicholas WT, Calverley PMA, Lee A, et al. Long-acting inhaled anticholinergic therapy improves sleeping oxygen saturation in COPD. Eur Respir J 2004; 23: 825-831.

65 Ryan S, Doherty LS, Rock C, et al. Effects of salmeterol on sleeping oxygen saturation in chronic obstructive pulmonary disease. Respiration 2010; 79: 475-481.

66 Aubier M, De Troyer A, Sampson M, et al. Aminophylline improves diaphragmatic contractility. N Engl J Med 1981; 305: 249-252.

67 Mulloy E, McNicholas WT. Theophylline improves gas exchange during rest, exercise, and sleep in severe chronic obstructive pulmonary disease. Am Rev Respir Dis 1993; 148: 1030-1036.

68 Mulloy E, McNicholas WT. Theophylline in obstructive sleep apnea. A double-blind evaluation. Chest 1992; 101: 753-757.

69 Connaughton JJ, Douglas NJ, Morgan AD, et al. Almitrine improves oxygenation when both awake and asleep in patients with hypoxia and carbon dioxide retention caused by chronic bronchitis and emphysema. Am Rev Respir Dis 1985; 132: 206-210.

70 Smith PL, Haponik EF, Allen RP, et al. The effects of protriptyline in sleep-disordered breathing. Am Rev Respir Dis 1983; 127: 8-13.

71 Sériès F, Marc I, Cormier Y, et al. Long-term effects of protriptyline in patients with chronic obstructive pulmonary disease. Am Rev Respir Dis 1993; 147: 1487-1490.

72 Roth T. Hypnotic use for insomnia management in chronic obstructive pulmonary disease. Sleep Med 2009; 10: 19-25.

73 Steens RD, Pouliot Z, Millar TW, et al. Effects of zolpidem and triazolam on sleep and respiration in mild to moderate chronic obstructive pulmonary disease. Sleep 1993; 16: 318-326.

74 Kryger M, Roth T, Wang-Weigand S, et al. The effects of ramelteon on respiration during sleep in subjects with moderate to severe chronic obstructive pulmonary disease. Sleep Breath 2009; 13: 79-84.

75 Kapella MC, Herdegen JJ, Perlis ML, et al. Cognitive behavioral therapy for insomnia comorbid with COPD is feasible with preliminary evidence of positive sleep and fatigue effects. Int J Chron Obstruct Pulmon Dis 2011; 6: 625-635.

76 Lloyd-Owen SJ, Donaldson GC, Ambrosino N, et al. Patterns of home mechanical ventilation use in Europe: results from the Eurovent survey. Eur Respir J 2005; 25: 1025-1031.

77 Schönhofer B, Köhler D. Effect of non-invasive mechanical ventilation on sleep and nocturnal ventilation in patients with chronic respiratory failure. Thorax 2000; 55: 308-313.

78 Elliott MW, Mulvey DA, Moxham J, et al. Domiciliary nocturnal nasal intermittent positive pressure ventilation in COPD: mechanisms underlying changes in arterial blood gas tensions. Eur Respir J 1991; 4: 1044-1052.

79 Goldstein RS, De Rosie JA, Avendano MA, et al. Influence of noninvasive positive pressure ventilation on inspiratory muscles. Chest 1991; 99: 408-415.

80 Meecham Jones DJ, Paul EA, Jones PW, et al. Nasal pressure support ventilation plus oxygen compared with oxygen therapy alone in hypercapnic COPD. Am J Respir Crit Care Med 1995; 152: 538-544.

81 Renston JP, DiMarco AF, Supinski GS. Respiratory muscle rest using nasal BiPAP ventilation in patients with stable severe COPD. Chest 1994; 105: 1053-1060.

82 Díaz-Lobato S, Alises SM, Rodríguez EP. Current status of noninvasive ventilation in stable COPD patients. Int J Chron Obstruct Pulmon Dis 2006; 1: 129-135.

83 Casanova C, Celli BR, Tost L, et al. Long-term controlled trial of nocturnal nasal positive pressure ventilation in patients with severe COPD. Chest 2000; 118: 1582-1590.

84 Clini E, Sturani C, Rossi A, et al. The Italian multicentre study on noninvasive ventilation in chronic obstructive pulmonary disease patients. Eur Respir J 2002; 20: 529-538.

85 Jones SE, Packham S, Hebden M, et al. Domiciliary nocturnal intermittent positive pressure ventilation in patients with respiratory failure due to severe COPD: long-term follow up and effect on survival. Thorax 1998; 53: 495-498.

86 Budweiser S, Jörres RA, Riedl T, et al. Predictors of survival in COPD patients with chronic hypercapnic respiratory failure receiving noninvasive home ventilation. Chest 2007; 131: 1650-1658.

87 McEvoy RD, Pierce RJ, Hillman D, et al. Nocturnal non-invasive nasal ventilation in stable hypercapnic COPD: a randomised controlled trial. Thorax 2009; 64: 561-566. 